\title{
Neuroinmunological response in HIV-associated Guillain-Barre syndrome: A case report ${ }^{*}$
}

\author{
Ivonne M. Iglesias-González ${ }^{1}$, Alberto J. Dorta-Contreras ${ }^{1 \#}$, Bárbara Padilla-Docal ${ }^{1}$, \\ Maylin Victoria-Garcia ${ }^{1}$, Ricardo Junco-Calzadilla ${ }^{2}$ \\ ${ }^{1}$ Faculty of Medical Sciences, “Dr Miguel Enríquez” Central Laboratory of Cerebrospinal Fluid (LABCEL), Havana Medical Sci- \\ ences University, Havana City, Cuba \\ ${ }^{2}$ Raúl Gómez García Hospital, Havana City, Cuba \\ Email: iigonzalez@infomed.sld.cu, \#吕orta@infomed.sld.cu, barbara.padilla@infomed.sld.cu, maylin.victoria@infomed.sld.cu, \\ labcel@infomed.sld.cu
}

Received 3 October 2011; revised 2 November 2011; accepted 15 November 2011

\begin{abstract}
Introduction: Human immunodeficiency virus infection is associated with several different types of peripheral neuropathy: distal predominantly sensory axonal polyneuropathy, like Guillain Barre syndrome. Case Presentation: A 55-year-old Caucasian woman with Human immunodeficiency virus infection, diagnosed with Guillain Barre syndrome was studied. Serum and CSF immunoglobulin G and Albumin levels were quantified by using an immunodiffusion technique. She had preceding viral symptoms. The clinical diagnosis of the illness in this patient was paresthesias or sensory loss, tendon reflexes. It was also observed cranial nerves abnormalities, acute motor and sensory axonal neuropathy and ophthalmoplegia. The mean CD4 count was $367 / \mathrm{mm}^{3}$, CSF white blood cell $25 \mathrm{wbc} / \mathrm{mm}^{3}$. The serum sodium concentration was significantly low $(133 \mathrm{mmol} / \mathrm{L})$. She had a respiratory compromise as a result of their neuropathy and developed congestive heart failure and hypotension and died of a cardiac arrest. The neuroimmunological response described by our patient was a blood/CSF barrier dysfunction without IgG intrathecal synthesis. Conclusion: There is no doubt that this study is of great importance because will help clinicians increase their knowledge of the immune response in patients with this autoimmune disorder on the basis of this case report, in which, for first time, could be seen the neuroimmunological response through the reibergram in a patient with HIVassociated Guillain-Barre syndrome.
\end{abstract}

Keywords: Guillain Barre; Human Immunodeficiency Virus; Neuroimmunological Response, Blood/CSF

\footnotetext{
"Competing interests: The authors declare that they have no competing interests.

${ }^{\#}$ Corresponding author.
}

Barrier

\section{INTRODUCTION}

Human immunodeficiency virus (HIV) infection is associated with different types of peripheral neuropathy: distal predominantly sensory axonal polyneuropathy, like Guillain Barre syndrome; mononeuritis multiplex; polyradiculopathy; acute or chronic inflammatory demyelinating polyneuropathies and toxic neuropathies caused by therapeutic drugs [1-3]. The association of Guillain Barre syndrome with HIV infection has been noted soon after the beginning of the HIV infection epidemic. The initial reports described it as typically occurring early in the course of HIV infection or even at the time of seroconversion [4].

Other than a commonly seen cerebrospinal fluid (CSF) pleocytosis, HIV-associated Guillain Barre syndrome is similar to that seen in non-HIV infected patients [5]. Many reviews suggest that Guillain Barre syndrome occurs early in HIV infection with high CD4 counts (>500) $[2,3]$. There is much evidence to support an immunological basic for this disease. The prevailing opinion is that demyelization in Guillain Barre syndrome is seconddary to a cellular mediated immune response directed against a component of peripheral myelin. Humoral immunity has also been found to be altered in Guillain Barre syndrome and may contribute to the pathogenesis of this disorder. The most important laboratory finding in Guillain Barre syndrome is an elevated CSF protein content without a pleocytosis [6,7].

The most typical neuroimmunological response in patients with Guillain Barre syndrome is the blood barrier dysfunction, it can be valued easily with the reibergram or CSF/serum quotient graphs [1,2]. There are diagrams that analyze in an integrated way both the function of the blood-CSF barrier and intrathecal protein synthesis, to 
aid in the diagnosis of central nervous system (CNS) diseases associated with specific patterns of immunoglobulin $[7,8]$. The diagnosis is performed by taking CSF and serum simultaneously in order to quantify Al- bumin and immunoglobulin G (IgG). Albumin is consid- ered a marker protein which is synthesized in the liver. It is found in CSF due to passage through the blood-CSF barrier $[9,10]$.

The objective of the present paper is to show the neuroimmunological response through the reibergram in a patient with HIV-associated Guillain Barre syndrome.

\section{CASE PRESENTATION}

Our patient was a 55-year-old Caucasian woman with HIV infection, diagnosed with Guillain Barre syndrome who was admitted to the hospital in June 2011. Guillain Barre syndrome neuroimmunological diagnosis was performed according to the protocol described in an earlier publication [9]. For all measurements, aliquots of serum were frozen and kept at $20^{\circ} \mathrm{C}$ for further analysis.

Serum and CSF levels of IgG and Albumin were quantified by an immunodiffusion technique using NOR and LC Partigen immunoplates (Siemens, Marburg).

The patient had other illnesses before her primary disease. She had preceding viral symptoms, upper respiratory symptoms, headache, malaise, conjunctivitis, myalgias and dizziness typical of acute primary HIV infection.

The clinical diagnosis of the illness in this patient was paresthesias or sensory loss, tendon reflexes. It was also observed cranial nerves abnormalities, acute motor and sensory axonal neuropathy and ophthalmoplegia, ataxia, is the most striking of several related conditions.

At the moment of her admittance the mean CD4 count was 367/ $\mathrm{mm}^{3}$ (range 55 - 800). Guillain Barre syndrome was not the first symptom of HIV infection in this patient. CSF white blood cell (WBC) was $25 \mathrm{wbc} / \mathrm{mm}^{3}$. The serum sodium concentration was significantly low (133 $\mathrm{mmol} / \mathrm{L}$ ), hyponatraemia. The serum sodium concentration was estimated by the use of an ion-selective electrode in diluted serum (normal range 136 - $146 \mathrm{mmol} / \mathrm{L}$ ). Validation of sodium concentration by ion-selective elec- trode technique in whole blood specimens was no routinely performed.

She had a respiratory compromise as a result of their neuropathy and was intubated for respiratory support and developed congestive heart failure and hypotension and died of a cardiac arrest 18 days after onset of symptoms at the Hospital's Intensive Care.

The concentrations of IgG and Albumin in blood and CSF are shown in Table 1. The normal value of each of these serum and CSF proteins was reported [10]. Blood and CSF profiles are shown in Table 2.

Results of IgG and Albumin in serum and SCF quantification were plotted on a Reibergram and shown in Figure 1. Our patient had a blood barrier dysfunction and there was no IgG intrathecal synthesis.

\section{DISCUSSION}

The prevailing opinion is that demyelination in Guillain Barre syndrome is secondary to a cellular-mediated immune response directed against a component of peripheral myelin. Humoral immunity has also been found to be altered in Guillain Barre syndrome and may contribute to the pathogenesis of the disorder. The most important laboratory finding in Guillain Barre syndrome is an elevated CSF protein content with a blood/CSF barrier dysfunction without immunoglobulin intrathecal synthesis [10].

A mild CSF pleocytosis in Guillain Barre syndrome suggests HIV infection [11], like it occurred in our case. Though CSF pleocytosis may suggest HIV infection, lack of pleocytosis does not exclude HIV infection. Since CSF pleocytosis has been noted in asymptomatic HIVpositive people, CSF pleocytosis may not always be related to Guillain Barre syndrome [12].

Cornblath et al. also noted that neurologically asymptomatic patients with HIV infection may have a CSF pleocytosis; the pleocytosis does not persist with signs of Guillain Barre syndrome, as would be expected if the pleocytosis were related solely to HIV infection and not to Guillain Barre syndrome [13].

The Albumin CSF/serum concentration quotient has been recognized as a suitable parameter to characterize

Table 1. Mean value, range in CSF and serum for albumin and IgG levels.

\begin{tabular}{|c|c|c|c|c|c|c|}
\hline & Age, years & Serum IgG (g/l) & CSF IgG (mg/l) & Serum Alb (g/l) & CSF Alb (mg/l) & $\mathrm{Q}\left(\mathrm{CSF} /\right.$ serum $\left.\times 10^{-3}\right)$ \\
\hline & & & & & & Q Alb $7.8 \times 10^{-3}$ \\
\hline \multirow[t]{3}{*}{ Patient } & 55 & 1.18 & 5.16 & 14.13 & 109.6 & \\
\hline & & & & & & $Q \operatorname{IgG} 4.4 \times 10^{-3}$ \\
\hline & & & & & & Q Alb mean $4.5 \times 10^{-3^{*}}$ \\
\hline \multirow[t]{2}{*}{ Normal values } & & 7 to 18 & 10 to 40 & 35 to 45 & 110 to 350 & \\
\hline & & & & & & Q IgG mean $2.05 \times 10^{-3}$ \\
\hline
\end{tabular}

IgG, immunoglobulin G; Alb, Albumin; Notes: Q = quotient (CSF/Serum); ${ }^{*}$ Mean value between ages 5 - 60 years old. 
Table 2. Blood and CSF profiles.

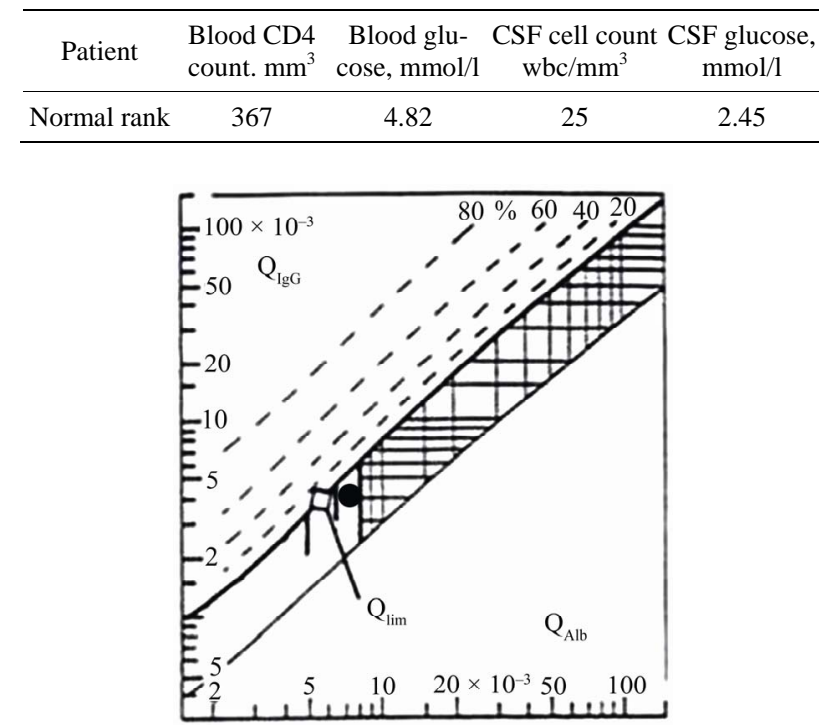

The upper hyperbolic curve (thick line) represents the discrimination line between brain-derived and bloodderived protein. Values above this upper line represent intrathecal IgG synthesis. The dashed hyperbolic lines indicate the extent of intrathecal synthesis as intrathecal fractions with $20 \%, 40 \%, 60 \%$, and $80 \%$ of the measured total immunoglobulin concentration in CSF, with reference to the discrimination line as $0 \%$ intrathecal synthesis. The limit of the reference range for Q Albumin between normal and increased CSF protein concentrations due to blood-CSF barrier dysfunction is indicated by the age-dependent vertical lines at $\mathrm{Q}$ Albumin $5.5 \times 10^{-3}$ (up to 15 years), at Q Albumin $6.5 \times 10^{-3}$ (up to 40 years), and at Q Albumin $8 \times 10^{-3}$ (up to 60 years). The black point represents the data from the patient suffering from GBS and HIV; notice that our patient had the IgG value in the normal range, indicating no intrathecal IgG synthesis, however there is blood barrier dysfunction.

Figure 1. CSF/serum quotient diagram for IgG or Reibergram.

the individual variables of the blood-CSF barrier function, which has been introduced as a suitable reference for the most sensitive evaluation of other plasma protein in CSF, owing to the reduction of individual variables [14].

Reibergrams or CSF/serum quotient graphs are diagrams that analyze in an integrated way both the function of the blood-CSF barrier and intrathecal protein synthesis, to aid in the diagnosis of CNS diseases associated with specific patterns of immunoglobulin response [13].

The most typical neuroimmunological response described in Guillain Barre syndrome is the CSF/blood barrier dysfunction which may be easily valued with a reibergram [6]. Also, generally in this disorder there isn't IgG intrathecal synthesis although it has communicated several exceptions. In our case we could appreciate this typical pattern.

\section{CONCLUSIONS}

There is no doubt that this study is of great importance because will help clinicians increase their knowledge of the immune response in patients with this autoimmune disorder on the basis of this case report, in which, for first time, could be seen the neuroimmunological response through the reibergram in a patient with HIVassociated Guillain-Barre syndrome.

Guillain Barre syndrome in a HIV patient could be a fatal combination in patients like this one in which an opportunistic biological agent accelerated de natural course of the disease.

\section{CONSENT}

Written informed consent was obtained from the patient's family for publication of this case. Copy of the written consent is available for review by the Editor-inChief of this journal.

\section{AUTHORS' CONTRIBUTIONS}

IIG and AJDC designed and coordinated the study and drafted the manuscript. BPD participated in its design and reviewed the clinical profiles of the patients. MVG helped in the manuscript translation. RJC helped with protein analysis and in drafting the manuscript. All authors read and approved the final manuscript.

\section{ACKNOWLEDGEMENTS}

The authors thank Manuel Rodriguez for technical assistance.

\section{REFERENCES}

[1] Brannagan, T.H., McAlarney, T. and Latov, N. (1998) Peripheral neuropathy in HIV-1 infection. In: Latov, N., Wokke, J.H.J. and Kelly, J.J. Eds., Immunological and Infectious Diseases of the Peripheral Nerves, Cambridge University Press, Cambridge, 285-307.

[2] Cornblath, D.R. and McArthur, J.C. (1995) Peripheral neuropathies in human immunodeficiency virus type 1 infection. In: Asbury, A.K. and Thomas, P.K. Eds., Peripheral Nerve Disorders, Butterworth, Oxford, 223-237.

[3] Simpson, D.M. and Tagliati, M. (1994) Neurologic manifestations of HIV infection. Annal of Internal Medicine, 121, 769-785.

[4] Cornblath, D.R., McArthur, J.C., Kennedy, P.G.E., Witte, A.S. and Griffin, J.W. (1987) Inflammatory demyelinating peripheral neuropathies associated with human T-cell lymphotropic virus type III infection. Annals of Neurology, 21, 320-340. doi:10.1002/ana.410210107

[5] Asbury, A.K. (1981) Diagnostic consideration in GuillainBarré syndrome. Annals of Neurology, 9, 1-5. doi:10.1002/ana.410090703

[6] Dorta-Contreras, A.J. (1999) Reibergramas: Elemento e- 
sencial en el análisis inmunológico del líquido cefalorraquídeo. Revista de Neurologia, 28, 996-998.

[7] Dorta-Contreras, A.J. and Silva-Somoza, R. (1996) Neuroimmunological findings in Guillain-Barré syndrome, multiple sclerosis and idiopathic polyneuropathies. Molecular and Chemical Neuropathology, 28, 279-284. doi:10.1007/BF02815233

[8] Reiber, H. (1994) The hyperbolic function: A mathematical solution of the protein flux/CSF flow model for blood CSF barrier function. Journal of the Neurological Sciences, 126, 243-245. doi:10.1016/0022-510X(94)90283-6

[9] Dorta-Contreras, A.J. (2006) Diagnóstico neuroinmunológico del síndrome de Guillain-Barré. Revista de Neurologia, 43, 640.

[10] Reiber, H. (1994) Flow rate of cerebrospinal fluid (CSF): A concept common to normal blood CSF barrier function and to dysfunction in neurological diseases. Journal of the Neurological Sciences, 122, 189-203. doi:10.1016/0022-510X(94)90298-4
[11] Centers for Disease Control and Prevention (1992) 1993 revised classification system for HIV infection and expanded surveillance case definition for AIDS among adolescents and adults. Morbidity and Mortality Weekly Report, 41, 1-19.

[12] Nishijima, T., Tsukada, K., Oka, S., et al. (2011) Antiretroviral therapy for treatment-naïve chronic HIV-1 infection with an axonal variant of Guillain-Barré syndrome positive for anti-ganglioside antibody: A case report. Internal Medicine, 50, 2427-2429.

[13] Cornblath, D.R., McArthur, J.C., Parry, G.J.G. and Griffin, J.W. (1993) Peripheral neuropathies in human immunodeficiency virus infection. In: Dyck, P.J., Thomas, P.K., Griffin, J.W., Low, P.A. and Poduslo, J.F., Eds., Peripheral Neuropathy, 3rd Edition, Saunders, Philadelphia, 1343-1345.

[14] Asbury, A.K., Arnason, B.G. and Adams, R.D. (1969) The inflammatory lesion in idiopathic polyneuritis. Its role in pathogenesis. Medicine, 48, 173-215. 